\title{
Pauwels screw combined inverted triangle cannulated screws for the treatment of Pauwels type-III femoral neck fracture:- a new surgical method based on the morphology of the fracture
}

\section{Gang Xue ( $\nabla$ xg1582281142@163.com )}

Wannan Medical College

\section{Siting Chen}

Wannan Medical College

Maosheng Zhou

Wannan Medical College

Zhengyu Wang

Wannan Medical College

Liangliang Wan

Wannan Medical College

JiaBing Xie

Wannan Medical College

Min Yang

Wannan Medical College

Research article

Keywords: Pauwels type-III femoral neck fracture,Internal fixtion,Pauwels screw, Inverted triangle cannulated screws, Morphologyical types.

Posted Date: July 2nd, 2020

DOl: https://doi.org/10.21203/rs.3.rs-38297/v1

License: (c) (i) This work is licensed under a Creative Commons Attribution 4.0 International License. Read Full License 


\section{Abstract}

Background: Femoral neck fracture is a serious injury in adults with significant functional consequences.Internal fixtion is an established treatment for femoral neck fractures in young adult patients .However, the risk of complications following fixation has plague orthopedists for decades. In all femoral neck fracture ,Pauwels type-III femoral neck fractures with a large vertical shear force were more likely to cause clinical failure.The aim of this study is to intruduce a new surgical technique for the treatment of this fracture according to the morphology of the fracture using the combination of Pauwels screw and inverted triangle cannulated screws(PSTCS)『and report the clinical effects in a prospective cohort of 14 patients.

Methods: From June 2017 to June 2019, a total of 14 patients (8 males and 6 females ) with Pauwels type-III femoral neck fracture underwent the surgical treatment of PSTCS according to the morphologyical types of fracture in clinical operation in our department. Patients were allowed to perform contraction exercises of quadriceps femoris on postoperative day1, passive flexion and extension exercises postoperative day 2 onwards, and active flexion and extension exercises postoperative day 7 onwards. Patients were allowed for partial-weight bearing walking postoperative 1month onwards with axillary crutches. After one month AP and lateral radiograph were taken. Full ambulation was permitted until the presence of radiological consolidation.Intraoperative (Operative duration,Blood loss),postoperation(Fracture union time,The Harris Hip Score,Visual analog score) were record for comparison.

Results: All patients were followed up for at least 12 months. In the end point of the follow up ,12 of the 14 patients $(85.7 \%)$ with pauwels type-III femoral neck fractures had achieved satisfactory hip function. Operative duration was a mean of $49.5 \pm 10.4$ minutes. Intraoperative blood loss was between 26 and 110 $\mathrm{ml}$ (mean, $44.4 \pm 20.6 \mathrm{ml}$ ) and without intraoperative blood transfusion. The Harris Hip Score was between

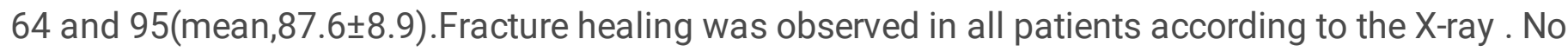
internal fixation was loosened or screw cut-out in the follow-up period.The femoral head necrosis was observed in 1 patient in this study. In addition, there was no obvious fracture displacement, femoral neck shortening, or hip varus.

Conclusions: The study showed that PSTCS can provide a satisfactory clinical outcomes for treatment of pauwels type-III femoral neck fractures.Most patients (85.7\%) achieved good or excellent results with the use of PSTCS, which appears to be a reliable method and provides another choice for this problematic fracture. The new internal fixation method of PSTCS can provide mechanical stability and promote fracture healing

\section{Background}

Femoral neck fracture is a serious problem in young adults caused by high-energy trauma, such as roadtraffic accidents ,sports injuries and falls from heights ${ }^{1}$.Although the treatment for this uncommon but 
clinically important fracture improving rapidly, The osteonecrosis rate in the femoral head is still as high as $15 \%-30 \%$, and the nonunion rate as high as $10 \%-30 \%^{2}$. Young femoral neck fracture patients are particularly at risk for experiencing complications, and suffer from re-operations. It continues to be unsolved fractures, and the guidelines for management are still evolving. Internal fixtion is a common method for the treatment of the femoral neck fractures in young adult patients, which can preserve the native femoral head and regain the natural structure and biomechanics ${ }^{1}$.In contrast, primary arthroplasty is favored for elderly patients.Although great progress has been made in the therapeutic field,the risk of complications following fixation of the femoral head has hardly changed in the past few decades,for example,the collapse of the femoral head, fracture nonunion, etc. ${ }^{3}$.It still represents a great challenge for orthopedists.

The pauwels classification has been used worldwide for measuring the shear force by using the angle of the fracture line compared with a horizontal line over the femoral head since first described in 1935. In this classification, femoral neck fractures are divided into 3 types type I: up to 30 degrees, type II: between 30 and 50 degrees, and type III: 50 degrees and more ${ }^{4}$. According to the research of Hulth,Increasing shear forces with increasing angle leads to more fracture instability, which increased possibility of loss of reduction and non-union ${ }^{5}$. Young adults with Type-III femoral neck fractures hited by high-energy trauma are more vulnerable to internal fixation failure, nonunion and avascular necrosis ${ }^{6-8}$.

Although Anatomical reduction and firm internal fixation are the main actions required to achieve a good outcome for type-III fracture,the appropriate thera-peutic schedule for these injuries is still controversial ${ }^{9}$ .Comparatively,multiple cannulated lag screws have the advantage of a relatively minimally invasive technique, shorter operative time, and sufficient fix-ation, which is preferred by most surgeons.Closed reduction and internal fixation by three cannulated screws is an accepted method with good results for the surgical treatment of Pauwels type-I and type-II fractures.Due to type-III femoral neck fracture has its own peculiar biomechanics distinct from other types,the frequency of complications such as nonunion and femoral head necrosis incidence was high with the treatment of traditional inverted triangle cannulated screw configuration.Pauwels type-III femoral fracture has a large vertical shear force.Conventional internal fixations are not sufficient to provide satisfactory stability,which prompted us to find a better surgical method to address this problem.

Pauwels screw which was inserted perpendicularly to the fracture line has higher internal fixation stability, especially anti-shear force capacity, so it has been paid increasingly more attention in clinical practice of type-III femoral neck fracture ${ }^{10}$. Hawks demonstrated that the technique using a screw perpendicular to the fracture plane can signficantly improve mechanical performance compared with the traditional technique ${ }^{11}$.Meanwhile,innovative configurations with four cancellous screws have been proposed by Kauffman ,which has a good mechanical properties in the femoral neck fracture with posterior comminution ${ }^{12}$. Based on previous studies and our experience, we describe a new surgical technique for the treatment of Pauwels type-III femoral neck fracture,which is the combination of Pauwels screw and 
inverted triangle cannulated screws(PSTCS), and represent the clinical outcomes in 14 patients after treatment with using this technique in this study.

\section{Materials And Methods}

From June 2017 to June 2019, a total of 14 patients underwent surgical treatment for Pauwels type-III femoral neck fracture in our level I trauma center (Table 1)Femoral neck fracture was suspected in patients whose Lower extremities are different in length and injury limb is in external rotation .Patients included who had acute trauma (within 24 hours of trauma), Ages younger than $60 \mathrm{y}$,there was no open fracture, all femoral neck fracture comes under pawels classification as type-III and were mentally stable and conscious. All of them had no surgical contraindication or any severe chronic systemic disease, were informed and agreed to consent of surgical protocol, follow up study and research. Patients who were excluded from study were pediatric patients, patients with old fractures, patients had a history of hip disease or hip surgery.All patients who met these criteria had the above-described surgical treatment performed.The study protocol and amendments were approved by the local institutional review boards. 
Table 1

Baseline Demographics of Participants and Follow-up

Results

\begin{tabular}{|l|}
\hline Age operative Blood Time Follow-Severe Visual Harris \\
Duration Loss union UP Compliction analog hip \\
NO Sex (Year) (min) (ml) (week) (Month) score score \\
\hline 1 M 5736351213 / 095 \\
2 F 5664321618 / 093 \\
3 F 5556421221 Avascular 364 \\
necrosis \\
4 M 4743541424 / 186 \\
5 F 5649401812 / 184 \\
6 F 4545341221 / 093 \\
7 F 5637421612 / 092 \\
8 M 5764261215 / 093 \\
9 M 5345321420 / 087 \\
10 F 5949371617 / 273 \\
11 M 4938361213 / 092 \\
12 M 4463481623 / 093 \\
13 M 3062541414 / 092 \\
14 M 42421101218 / 090 \\
\hline
\end{tabular}

The patient was placed supine on the radiolucent operation table under General anesthesia or epidural anesthesia.The foot of the injured lower extremity is fixed for further traction.meanwhile,The contralateral lower extremity is fixed with hip joint flexion, abduction, and external rotation and knee joint flexion ,which can get a proper lateral view of the injured extremity.Closed reduction of the femoral neck is performed with the patient using image intensification.Longitudinal traction is applied in line with the femur to restore the length of the injured lower limb while abducted position is maintained.the limb is then circumducted into an internal rotation and adduction position to reconstruct the femoral neck and to achieve an optimal reduction(Fig. 1). A prefect reduction can be obtained by closed means, as verified on both the AP and lateral fluoroscopic views ${ }^{13}$,otherwise,the minimally invasive Watson-Jones approach was adopted ${ }^{14}$

Following routine skin disinfection,the first guide wire were inserted according to the Morphology of the fracture line of pauwels type-III after reduction under the guideance of image intensification,which is the 
key to this surgical method.the Pauwels type-III femoral neck fracture basically present the following four Morphologyical types of fracture line in clinical operation(Fig. 2). Type A:Simple oblique fracture,Type B:Serrated fracture,TypeC: Simple comminuted fracture,Type D:Complex comminuted fracture.In this study,five patients were of the Morphologyical type A,two patients were of the Morphologyical type B,four patients were of the Morphologyical type C,and three patients were of the Morphologyical type D.For type $A$ and $B$,The first guide-wire was placed upper, inserted from the lateral aspect of the greater tro-chanter towards the femoral head through a small stab incision, which verticaled the fracture line on both the AP and lateral fluoroscopic views.For type $C$ and $D$,in addition to satisfying the perpendicular to the fracture plane, the first guide wires should be placed in the position of the anterior of the femoral neck,which is the supportable section for a apprepriate compression. Then,the rest three guide wires are inserted into the femoral neck in a parallel inverted triangle configuration (inferior, posterosuperior, an-terosuperior) with situation of the screws adjacent to the infe-rior (calcar) and posterior cortices .All the above manipulations should be performed gently to avoid fracture displacement.

The optimal reduction of the femoral neck fracture site is confirmed once more by the image intensifier.then after reaming under guidance of the guide wires, the Pauwels cannulated screw is firstly inserted into the femoral neck with appropriate compression to ensure no gaps between fractures.Pauwels cannulated screw is perpendicular to the fracture line on both the AP and lateral fluoroscopic views, which can resist the tremendous shear force of pauwels type-III fracture. The inserted orders of the remaining three screws were different according to the Morphologyical types of fracture line.For type A and B,as the conventional surgery procdure, the inverted triangle cannulated screws are inserted into the femoral neck. But for type $C$ and $D$,Due to the posteromedial comminution of the femoral neck,Priority is given to the anterior-superior screws to insert in the femoral neck .Subsequently,the remaining two cannulted screws were inserted in standard lag fashion.Finally,careful hemostasis, disinfection, and wound suture completed the surgery when the k-wires were pulled out and final fluoroscopic views are obtained.

Patients were allowed to perform contraction exercises of quadriceps femoris on postoperative day1, passive flexion and extension exercises postoperative day 2 onwards, and active flexion and extension exercises postoperative day 7 onwards. Patients were allowed for partial-weight bearing walking postoperative 1 month onwards with axillary crutches. After one month AP and lateral radiograph were taken. Full ambulation was permitted until the presence of radiological consolidation. Routine radiological and clinical assessment was performed every 1 month until the presence of radiological consolidation and then every 3 months thereafter (minimum time for follow up was one year to a maximum of two years) postoperatively. Fracture healing was considered after bony callus formation across the fracture line, faded fracture line on X-Ray accompanied with that patient had no local pain or tenderness, the ability to walk well without help.

We included intraoperative clinical outcome as operative duration (operative duration was defined as the time from initiation of the incision to the time when suture of the incision was finished), blood loss (recorded in $\mathrm{ml}$ by expected value and measured by the hemorrhage through the suction instruction in 
operation), fracture union time(Fracture union was defined as a visible callus bridging the fracture site present within 6 months of surgery ${ }^{15}$ ), and postoperative complications. Fracture displacement were evaluated with fluoroscopy 1 month postoperatively .The Harris Hip Score ${ }^{16}$ (The Harris hip score was considered excellent at 90 to 100 , good at 80 to 89 , fair at 70 to 79 , and poor at $<70$ ) and visual analog score were recorded for evaluation of hip function at the final follow-up.All patients'outcomes were assessed by the same two observers .

\section{Results}

Fourteen patients were included in the study ,11 patients trauma caused by road traffic accident(7 automobile accidents and 4 electric bicycle accidents), and the remaining 3 patients was caused by sudden fall.Five fractures occurred on the right side and nine on the left side,with the mean age of(50.4 \pm 8.1)(range 30-57 years).All patients were followed up for at least 12 months. Perfect reduction were achieved in all patients by closed reduction in this study .Operative duration was a mean of $49.5 \pm 10.4$ minutes. Intraoperative blood loss was between 26 and $110 \mathrm{ml}$ (mean, $44.4 \pm 20.6 \mathrm{ml}$ ) and without intraoperative blood transfusion. Fracture healing was observed in all patients. The mean time to radiographic fracture healing was $(14.0 \pm 2.1)$ weeks (range 12 to 18 weeks).The hip function was evaluated according to Harris Hip Score system ( $<70$ Poor, 70-79 fair ,80-89 Good, $>90$ excellent) at the final follow-up with the mean score was $87.6 \pm 8$.9.Twelve of the 14 patients $(85.7 \%)$ obtained satisfactory hip function. Thirteen of the 14 patients $(92.8 \%)$ believed that they had recovered preoperative function at the final follow-up.No internal fixation was loosened or screw cut-out in the follow-up period.the femoral head necrosis was observed in 1 patient in this study. In addition, there was no obvious fracture displacement and femoral neck shortening( measured as described by Zielinski et al ${ }^{17}$ ), or hip varus.

\section{Discussion}

Although pauwels type-III femoral neck fracture represents a relatively uncommon injury in the young pupulation, this type fracture is problematic and challenging ${ }^{18}$.It is characterized by a small compression load at the fracture, but a large shear stress and varus load.Given the machanical behavior of the Pauwels type-III fracture,the internal fixtion should withstand the large vertical shear forces to achieve stablility of the fracture.there is still no gold standard protocol on the optimal treatment of pauwels typeIII femoral neck fracture.Muiltiple cannulated screws has its own strengths, which is a relatively noninvasive procedure, shorter operative time,the relatively simpler surgical procedure and an acceptable less intra-operative visible blood loss.However, conventional parallel inverted triangle screws are thought to have poor biomechanical performance for the treatment of pauwels type-III femoral neck fracure .

Pauwels screw provide a new reference for the treatment of vertical femoral neck fracture.Filipov found that cross-divergent cancellous lag screws can significantly improve the fixation strength of the femoral neck fracture after follow-up of 207 patients in the 5 years period ${ }^{19}$.Some studies have shown that the stability of the femoral neck fracture can be significantly increased by use of a pauwels screw construct 
to fix vertically oriented femoral neck fracture ${ }^{11}$. In our study, All patients with pauwels type-III femoral neck fractures were fixed with the combination of Pauwels screw and inverted triangle cannulated screws.the configuration of PSTCS present good biomechanical stability.our results show that twelve of 14patients (85.7\%) obtained satisfactory hip function .the femoral head necrosis was observed in 1 patient in this study and the poor glycemic controlling maybe the potential cause of this outcome.No internal fixation failure was found in 14 patients in the follow-up period.

Proper anatomic reduction and stable internal fixation is the key to preserve the femoral head, avoiding avascular necrosis, nonunion, and femur shortening ${ }^{20}$.The Pauwels type-III femoral neck fractures in young adult patients are often the result of high-energy trauma. approximately $70 \%$ of the cases of the fracture are combined with communited fractures in the posterior-medial of the femoral neck,which may reduce postoperative fixation stability and increase the risk of complications such as nonunion and osteonecrosis ${ }^{21,22}$.moreover,Previous studies have shown that the risk of fracture instability increases significantly when the fracture area exceeded $25 \%$ of the posterior femoral neck regions ${ }^{23}$.Kauffman et al show that compared with femurs fixed with three screws, four screws have significantly higher resistance to axial and forward displacement, and the axial destructive force continues to decrease ${ }^{12}$.In our study,all 14 patients were treated with PSTCS according to the femoral neck fracture morphology,all patient achieved a stable fixation and did not experience re-displacement of the fracture after the operation during follow-up.The PSTCS can stabilize the fracture end in multiple planes ,placing a pauwels screw through the fracture line at a 90-degree angle may achieve optimal control of the shear force at the fracture end and effectively reduce the occurrence of postoperative internal failure rate.Besides this, in actual clinical practice,the relatively complete and supportable sections of the fracture ends are fixed preferentially.It also can reduce the incidence and degree of the femoral neck shortening and the rotation of femoral head caused by excessive compression of the screw in the comminuted fracture part. However,the effect of fracture morphology on internal fixation of Pauwels type-III femoral neck fracture is often overlooked by most Orthopedic surgeons in clinical work.Currently, Pauwels classification system is still the practical mainstay of femoral neck fracture characterization that help dictate appropriate treatment, although some scholars believe that Classification of intra-capsular hip fractures according to the Pauwels classification using the Pauwels angle is unreliable and its use should be avoided ${ }^{24}$.

For pauwels type III fractures,most clinicians only focus on the choice of internal fixation,but ignore the morphological differences of the fracture line and the and the associated surgical operation techniques ,especially the comminuted posterior-medial fracture of pauwels type-lll femoral neck fracure. Theoretically speaking, The final outcome of pauwels type-III femoral neck fracure depends not only on the surgical devices, but on surgical techniques. The morphology of the pauwels type-IIl fracure may detemine the orders of screw compression and the position in the femur neck. As we elaborated earlier,There are 4 types of patterns based on the morphology of pauwels type III fracture,which was found in our Clinical Work Practice.For type A and type B,pauwels cannulated screw was inserted firstly to press the fracture end and eliminate the fracture gap, which can maintain the femoral neck length, 
withstand vertical shear forces and achieve bone union during operation.For type $\mathrm{c}$ and type D,Comminuted fractures are mainly concentrated on the posterior-medial of the femoral neck. The anterior of the femoral neck is relatively complete. the pauwels screws and the anterior- upper screws can take advantage of this supportable section for a apprepriate compression,so pauwels screw and anteriorsuperior screw were inserted earlier than inferior screw and posterosuperior screw. In our study, the patients of pauwels type-III femoral neck fracure treated by PSTCS achieved good clinic results in this way.there was no obvious fracture displacement and femoral neck shortening. only one of the patients developed necrosis of the femoral head.

\section{Conclusion}

The operative treatment of pauwels type-III femur neck fracture is still a challenge even for an experienced surgeon. The study showed that PSTCS can provide a satisfactory clinical outcomes for treatment of this fracture.Most patients (85.7\%) achieved good or excellent results with the use of PSTCS, which appears to be a reliable method and provides another choice for this problematic fracture .Larger, randomized controlled studies comparing these operation method are needed to determine which treatment is associated with less complications and more consistent outcomes. This study should provide the rationale and our data can help inform sample- size calculations for such studies.

\section{Abbreviations}

PSTCS :Pauwels screw and inverted triangle cannulated screws

\section{Declarations}

\section{Acknowledgements}

Not applicable

\section{Authors' contributions}

M.Y and G.X participated in the recruitment, data collection, and analysis.All the authors contributed to the study design and drafting of the manuscript. All the authors read and approved the final manuscript.

\section{Funding}

This study was supported by Young and Middle-aged Project Fund Of Wannan Medical College (No.KY26260426) and Talent Introduction Plan of Yijishan Hospital,Wannan Medical college(No.YR201917)

\section{Availability of data and materials}


The datasets supporting the conclusions of this article are included within the article. The raw data can be requested from the corresponding author on reasonable request.

\section{Ethics approval and consent to participate}

The ethics committee of Yijishan Hospital of Wannan Medical College approved the development of this research study .written informed consent was obtained from all patient,which have been performed in accordance with the ethical standards of declaration of helsinki and its later amendments.

\section{Consent for publication}

All patients and caregivers consented to the publication of their personal data in this article

\section{Competing interests}

On behalf of all authors, the corresponding author states that there is no conflict of interest.

\section{References}

1. Lein T, Bula P, Jeffries J, Engler K, Bonnaire F. Fractures of the femoral neck. Acta Chir Orthop Traumatol Cech 2011;78(1):10-9.

2. Wu CC. Using biomechanics to improve the surgical technique for internal fixation of intracapsular femoral neck fractures. Chang Gung Med J 2010;33(3):241-51.

3. Schmidt AH, Swiontkowski MF. Femoral neck fractures. Orthop Clin North Am 2002;33(1):97-111, viii.

4. Bartonicek J. Pauwels' classification of femoral neck fractures: correct interpretation of the original. J Orthop Trauma 2001;15(5):358-60.

5. Hulth $A$. The inclination of the fracture surfaces and its relation to the rate of healing in femoral neck fractures. Acta Chir Scand 1961;121:309-14.

6. Liporace F, Gaines R, Collinge C, Haidukewych GJ. Results of internal fixation of Pauwels type-3 vertical femoral neck fractures. J Bone Joint Surg Am 2008;90(8):1654-9.

7. Protzman RR, Burkhalter WE. Femoral-neck fractures in young adults. J Bone Joint Surg Am 1976;58(5):689-95.

8. Zlowodzki M, Brink $\mathrm{O}$, Switzer J, et al. The effect of shortening and varus collapse of the femoral neck on function after fixation of intracapsular fracture of the hip: a multi-centre cohort study. $J$ Bone Joint Surg Br 2008;90(11):1487-94.

9. Luttrell K, Beltran M, Collinge CA. Preoperative decision making in the treatment of high-angle "vertical" femoral neck fractures in young adult patients. An expert opinion survey of the Orthopaedic Trauma Association's (OTA) membership. J Orthop Trauma 2014;28(9):e221-5.

10. Guimaraes JAM, Rocha LR, Noronha Rocha TH, et al. Vertical femoral neck fractures in young adults: a closed fixation strategy using a transverse cancellous lag screw. Injury 2017;48 Suppl 4:S10-S16. 
11. Hawks MA, Kim H, Strauss JE, et al. Does a trochanteric lag screw improve fixation of vertically oriented femoral neck fractures? A biomechanical analysis in cadaveric bone. Clin Biomech (Bristol, Avon) 2013;28(8):886-91.

12. Kauffman JI, Simon JA, Kummer FJ, et al. Internal fixation of femoral neck fractures with posterior comminution: a biomechanical study. J Orthop Trauma 1999;13(3):155-9.

13. Lindequist S, Tornkvist H. Quality of reduction and cortical screw support in femoral neck fractures. An analysis of 72 fractures with a new computerized measuring method. J Orthop Trauma 1995;9(3):215-21.

14. de Jong L, Klem T, Kuijper TM, Roukema GR. The minimally invasive anterolateral approach versus the traditional anterolateral approach (Watson-Jones) for hip hemiarthroplasty after a femoral neck fracture: an analysis of clinical outcomes. Int Orthop 2018;42(8):1943-48.

15. Huang TW, Hsu WH, Peng KT, Lee CY. Effect of integrity of the posterior cortex in displaced femoral neck fractures on outcome after surgical fixation in young adults. Injury 2011;42(2):217-22.

16. Harris WH. Traumatic arthritis of the hip after dislocation and acetabular fractures: treatment by mold arthroplasty. An end-result study using a new method of result evaluation. J Bone Joint Surg Am 1969;51(4):737-55.

17. Zielinski SM, Keijsers NL, Praet SF, et al. Femoral neck shortening after internal fixation of a femoral neck fracture. Orthopedics 2013;36(7):e849-58.

18. Singer BR, McLauchlan GJ, Robinson CM, Christie J. Epidemiology of fractures in 15,000 adults: the influence of age and gender. J Bone Joint Surg Br 1998;80(2):243-8.

19. Filipov O, Stoffel K, Gueorguiev B, Sommer C. Femoral neck fracture osteosynthesis by the biplane double-supported screw fixation method (BDSF) reduces the risk of fixation failure: clinical outcomes in 207 patients. Arch Orthop Trauma Surg 2017;137(6):779-88.

20. Ghayoumi P, Kandemir U, Morshed S. Evidence based update: open versus closed reduction. Injury 2015;46(3):467-73.

21. Caviglia HA, Osorio PQ, Comando D. Classification and diagnosis of intracapsular fractures of the proximal femur. Clin Orthop Relat Res 2002(399):17-27.

22. Scheck M. The significance of posterior comminution in femoral neck fractures. Clin Orthop Relat Res 1980(152):138-42.

23. Rawall S, Bali K, Upendra B, et al. Displaced femoral neck fractures in the young: significance of posterior comminution and raised intracapsular pressure. Arch Orthop Trauma Surg 2012;132(1):739.

24. van Embden D, Roukema GR, Rhemrev SJ, Genelin F, Meylaerts SA. The Pauwels classification for intracapsular hip fractures: is it reliable? Injury 2011;42(11):1238-40.

\section{Figures}




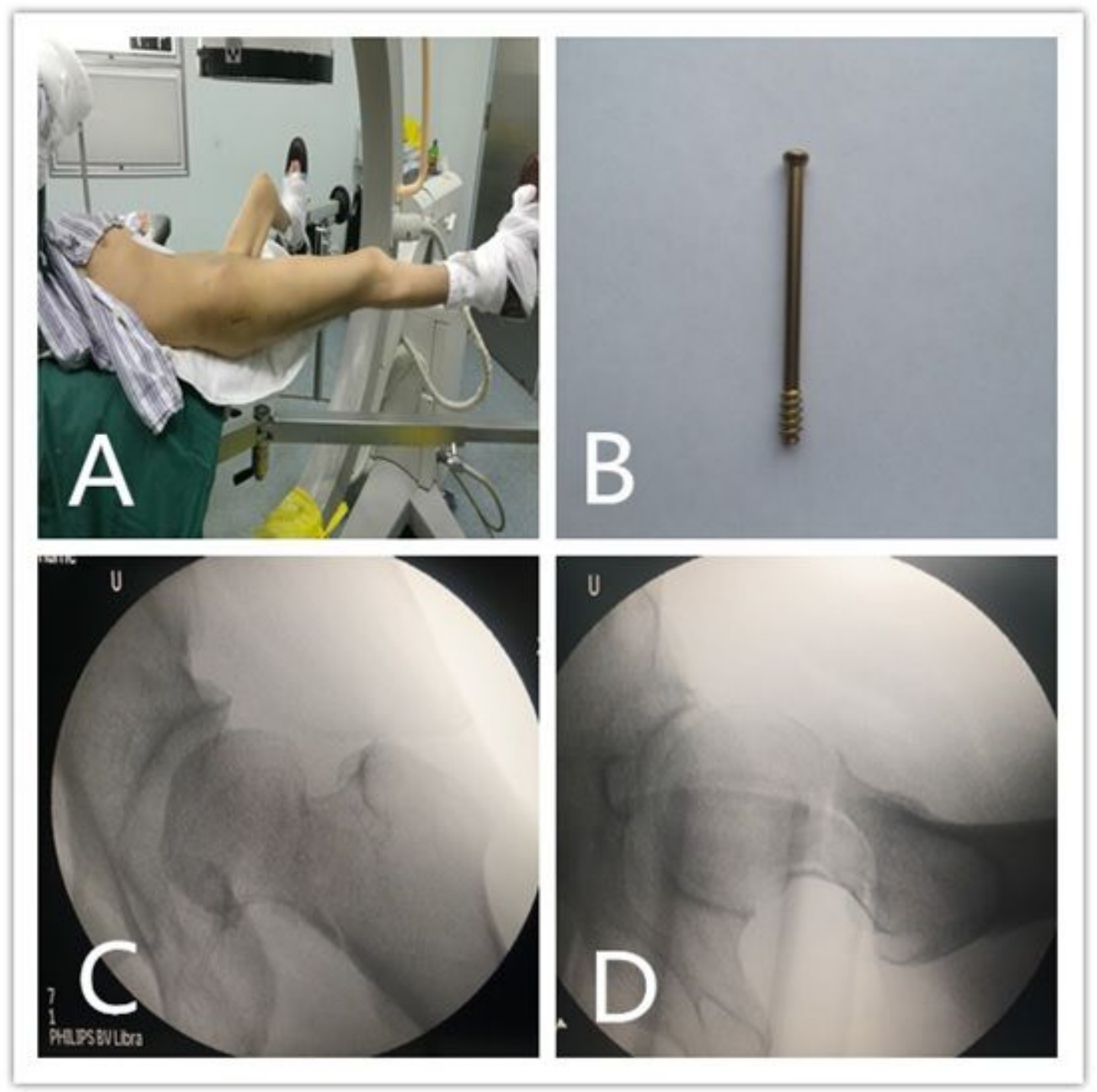

Figure 1

(A):Surgical posture.(B) Cannulated screws with a diameter of $7.3 \mathrm{~mm}$ used in the operation $\nabla$ from The

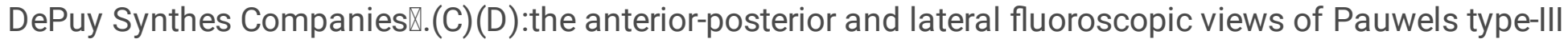
femoral neck fracture after colsed reduction during surgical operation. 


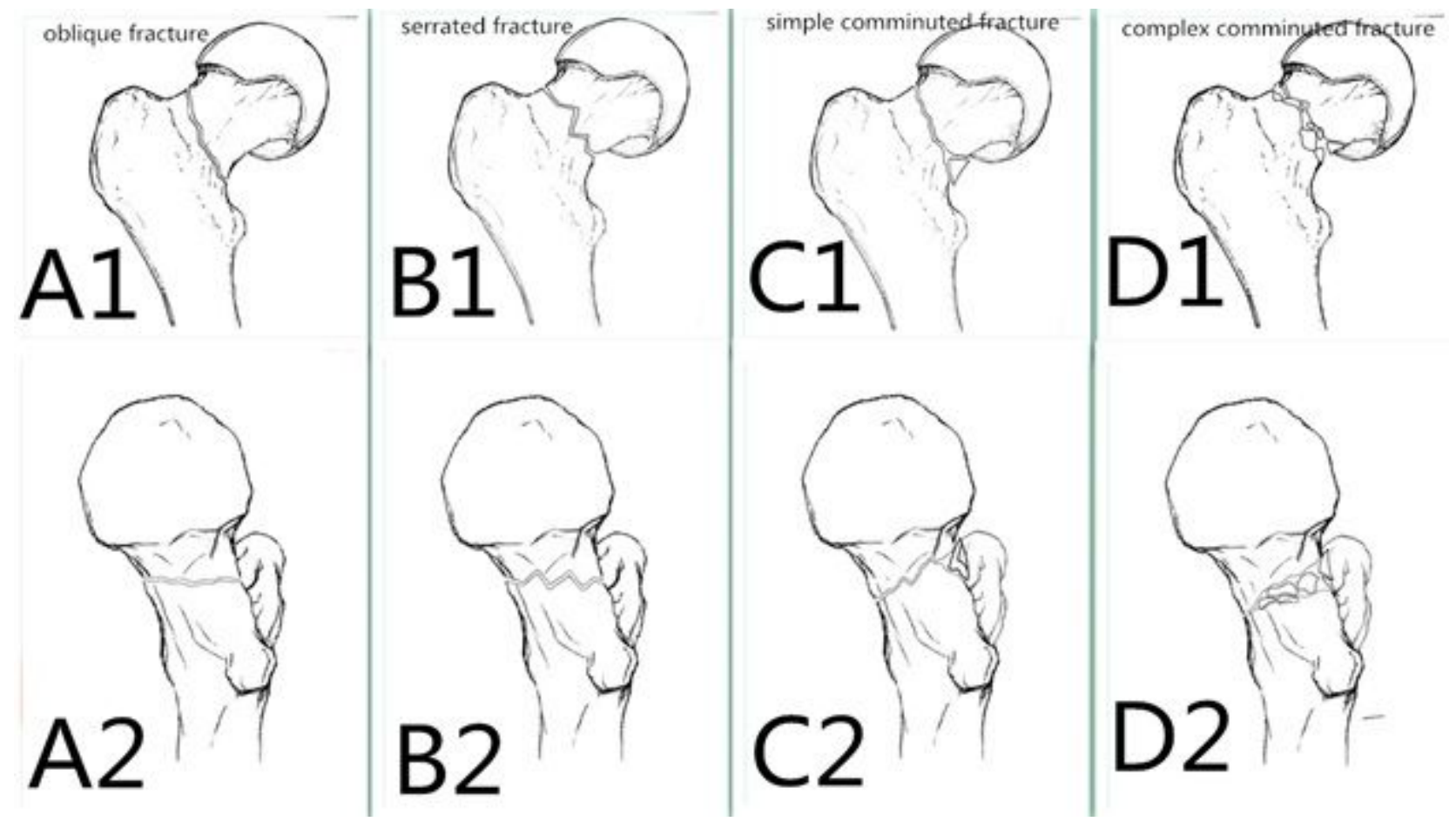

Figure 2

the morphological types of fracture lines in Pauwels type-lll femoral neck fractures .(A):simple oblique fracture.(B):serrated fracture.(C): simple comminuted fracture.(D): complex comminuted fracture.

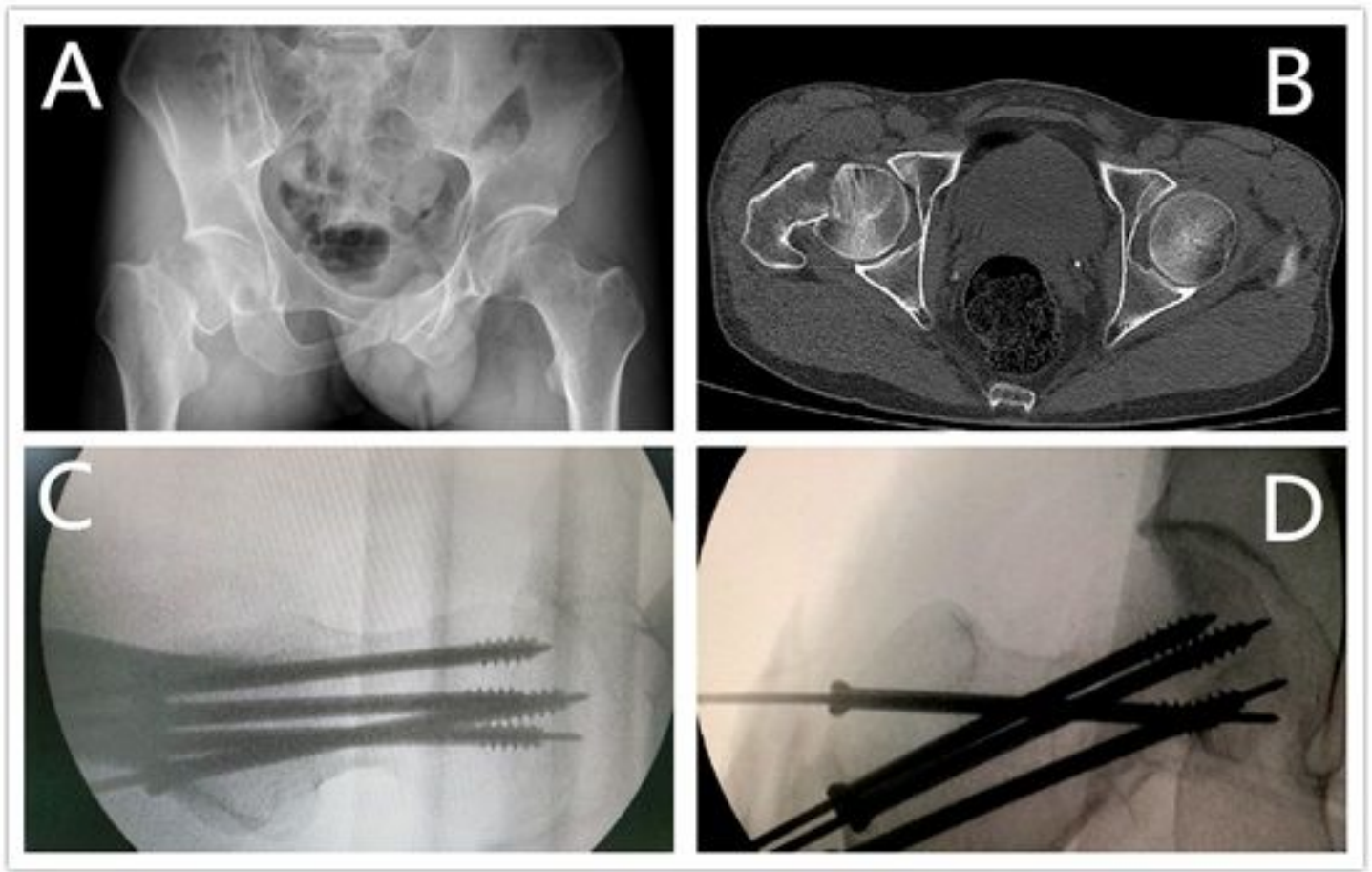

Figure 3

a 30 years old male patient,he had a Pauwels type-III femoral neck fracture of right lower limb in a traffic accidient.(A).X-ray of anterior-posterior showed that the fracture end is significantly displaced,with a pauwels angle of approximately 60 degrees.(B)CT scan showed comminuted fractures of posteromrdial 
femoral neck before operation.(C)(D)The x-ray showed that the Pauwels type-III femoral neck fracture got a satisfactory reduction.the pauwels screw was perpendicular to the fracture line on both the AP and lateral fluoroscopic views under the guideance of image intensification.

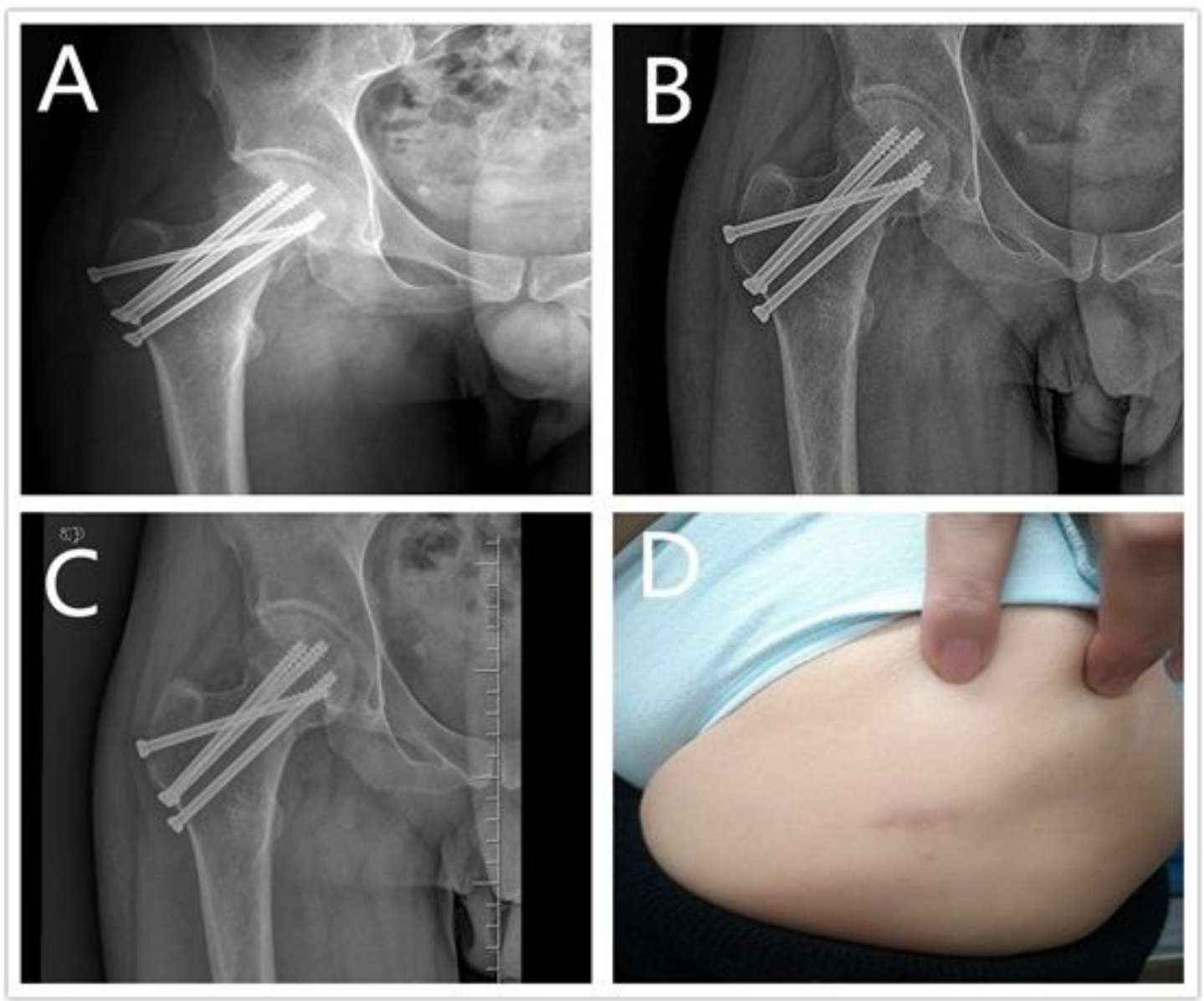

\section{Figure 4}

Postoperative follow-up in the same patient. (A)The fracture plane was stable without redisplacement but the fracture was still obvious at 12 weeks after operation . (B) A callus formation was seen around fracture site and the fracture line was faded on X-ray at 16 weeks after operation.(C).The internal fixtation is stable without failure and the pauwels type-3 femoral neck facture healed completely within 1 year after operation.(D).The insion size was small with a small scars 